\title{
Expression of reproductive hormone receptors and contraction-associated genes in porcine uterus during the estrous cycle
}

\author{
SUNG-MIN AN $^{1 *}$, SUN SUK KIM $^{2 *}$, JUN KIM $^{1}$, MEE-NA PARK $^{3}$, JAE-EON LEE $^{1}$, \\ SEONG KEUN CHO ${ }^{4}$, KYU-SUP LEE ${ }^{2}$ and BEUM-SOO AN ${ }^{1}$
}

\begin{abstract}
${ }^{1}$ Department of Biomaterials Science, College of Natural Resources and Life Science, Pusan National University, Miryang, Gyeongsangnam-do 50463; ${ }^{2}$ Department of Obstetrics and Gynecology, Biomedical Research Institute, Pusan National University School of Medicine, Pusan, Gyeongsangnam-do 49241; ${ }^{3}$ University of Ulsan College of Medicine, Seoul, Gyeonggi-do 05505; ${ }^{4}$ Department of Animal Science, College of Natural Resources and Life Science, Pusan National University, Miryang, Gyeongsangnam-do 50463, Republic of Korea
\end{abstract}

Received August 31, 2016; Accepted March 21, 2017

DOI: $10.3892 / \mathrm{mmr} .2017 .6518$

\begin{abstract}
Contraction of uterus tissue frequently occurs throughout the estrous cycle and is regulated by several endogenous factors, including estradiol, progesterone, luteinizing hormone, follicle-stimulating hormone, oxytocin (OXT) and contraction-associated proteins (CAPs). Contraction activity of uterus tissue according to the estrous cycle is important, due to the fact that it is directly associated with balanced implantation and stable pregnancy. However, few studies have examined the mechanism of uterus contraction activity in a porcine model. In the current study, porcine uterus tissue was separated into the follicular and luteal phases by histological analysis. To investigate regulation of contraction-associated factors according to the estrous cycle, mRNA and protein expression levels of reproductive hormonal receptors, including estrogen receptors, progesterone receptor and luteinizing hormone/choriogonadotropin receptor in addition to CAPs including OXT, OXT receptor (OXTR), hydroxyprostaglandin dehydrogenase 15-(NAD) and gap junction $\alpha-1$ protein, were examined in the porcine uterus according to the follicular
\end{abstract}

Correspondence to: Dr Beum-Soo An, Department of Biomaterials Science, College of Natural Resources and Life Science, Pusan National University, 50 Cheonghak-ri, Samrangjin-eup, Miryang, Gyeongsangnam-do 50463, Republic of Korea

E-mail: anbs@pusan.ac.kr

Dr Kyu-Sup Lee, Department of Obstetrics and Gynecology, Biomedical Research Institute, Pusan National University School of Medicine, Ami-Dong 1-Ga, Seo-Gu, Pusan, Gyeongsangnam-do 49241, Republic of Korea

E-mail: kuslee@pusan.ac.kr

${ }^{*}$ Contributed equally

Key words: porcine, uterus, estrous cycle, uterine contraction, contraction-associated proteins and luteal phases. For the results, hormonal receptors and CAPs were dynamically regulated depending on the estrous cycle. In conclusion, genes associated with uterine contraction and its regulatory hormonal receptors in the porcine uterus were differently regulated in the follicular and luteal phases, suggesting that these genes are critically involved in the remodeling and contraction of uterine tissue and may be required to modulate the physiological status of the uterus.

\section{Introduction}

The uterus is a female reproductive organ composed of a well-differentiated endometrium, a thick muscular myometrium, and an outer serosal layer (1). Marked progress has been made in understanding the physiology and pathophysiology of the endometrium, resulting in the development of important interventions for menstrual function, conception and contraception. Physiological functions of the endometrium are differentially regulated by steroid hormones and peptides depending on the estrous cycle (2). The mammalian estrous cycle, including follicular and luteal phases, is regulated by a positive and negative feedback system involving reproductive hormones that are manufactured and released from organs including the hypothalamus, pituitary and ovaries (3). In the follicular phase (also called proliferative phase), estradiol (E2) leads to lining of the uterus for development and proliferation of endometrium. Following uterus maturation, ovarian follicles secrete an increasing quantity of E2 to initiate development of a new layer of proliferative endometrium in the uterus in addition to provoking crypts in the cervix to produce rich cervical mucus (4). Following ovulation, E2 production is reduced while the corpus luteum begins to form and produce progesterone (P4), which serves an important role in implantation of blastocysts and support of early pregnancy in the endometrium. High concentrations of P4 have a negative effect on follicular growth by preventing secretion of follicle-stimulating hormone (FSH) and luteinizing hormone ( $\mathrm{LH})(5)$. This phase is called the secretory phase and corresponds to the luteal phase of 
the estrous cycle. If female mammals are not pregnant at the luteal phase, prostaglandin $\mathrm{F}_{2 \alpha}\left(\mathrm{PGF}_{2 \alpha}\right)$ is released from the endometrium, and the corpora lutea rapidly regresses and is degraded. In addition, P4 levels rapidly decline while FSH and LH gradually increases to stimulate ovarian follicle growth, which is known as the follicular phase. As ovarian follicles grow, E2 concentrations reach high levels and stimulate ovulation again. The estrous cycle in mammalian females repeats until pregnancy (6).

The uterus frequently contracts throughout the entire estrous cycle. Stable uterine contractile activity is an essential factor for maintenance of pregnancy in addition to appropriate timing of childbirth. By contrast, inappropriate uterine contraction leads to premature birth during pregnancy, which is currently recognized as one of the leading causes of perinatal death in the developed world. Premature birth in humans, defined as labor prior to 37 weeks gestation, occurs in $7-10 \%$ of all births, however, amounts for $>85 \%$ of all complications and mortality associated with child birth (7). Preterm labor likely represents a syndrome rather than a specific diagnosis, due to the fact that the causes are varied. It may reflect a breakdown in the mechanisms responsible for maintaining uterine quiescence (8). In mice and rats, $\mathrm{P} 4$ promotes myometrial relaxation and sustains pregnancy, whereas E2 increases uterine contractility during parturition $(9,10)$. P4 appears to induce relaxation of the myometrium by repressing expression of genes that encode factors collectively referred to as contraction-associated proteins (CAPs), which promote labor. Some important CAPs include oxytocin receptor (OXTR), prostaglandin (PG) metabolizing enzyme hydroxyprostaglandin dehydrogenase 15-(NAD) (HPGD), and gap junction $\alpha-1$ protein (GJA1) (11). A previous study described a novel pathway in which $\mathrm{P} 4$ coordinately represses expression of two critical CAP genes, specifically GJA1 (encodes a key gap-junction protein that synchronizes contractile activity) and OXTR (determines responsiveness of myometrial cells to OXT, a potent stimulator of contraction) (12). In contrast, E2 is known to upregulate expression of CAPs in the uterus and thereby, stimulates uterine contraction. Thus, correct balance between P4 and E2 is essential for uterine function and successful pregnancy. Although regulation of uterine contraction by steroid hormones during pregnancy and parturition has been studied, few reports have focused on this issue in non-pregnant animals. Furthermore, few studies have examined the porcine reproductive system according to the estrous cycle. It is important to understand the porcine reproductive system due to the fact that it is directly connected to the birth ratio of pigs, which is key in meat production processes.

In the present study, the expression levels of receptors corresponding with reproductive hormones and CAPs were examined according to the estrous cycle in the porcine uterus in order to improve the understanding of the physiological and endocrine circumstances of the porcine reproductive system.

\section{Materials and methods}

Reagents and chemicals. Rabbit anti- $\beta$-actin (no. 4967) was purchased from Cell Signaling Technology, Inc. (Danvers, MA, USA). Rabbit anti-estrogen receptor 1 (ESR1; sc-542), ESR2 (sc-8974), rabbit anti-progesterone receptor (PGR; sc-538), rabbit anti-luteinizing hormone/choriogonadotropin receptor (LHCGR; sc-25828) goat anti-OXTR (sc-8102) and rabbit anti-HPGD (sc-98907) antibodies were purchased from Santa Cruz Biotechnology, Inc. (Santa Cruz, CA, USA). Rabbit anti-GJA1 (ab11370) was purchased from Abcam (Cambridge, MA, USA). Mouse anti-OXT (MAB5296) was purchased from Merck Millipore (Darmstadt, Germany). Horseradish peroxidase (HRP)-conjugated anti-rabbit (sc-2313), anti-mouse (sc-2005) and anti-goat IgG (sc-2020) secondary antibodies were purchased from Santa Cruz Biotechnology, Inc.

Tissue preparations. Peripubertal crossbred gilts $(\mathrm{n}=20$, Eoband-dong, Gimhae-si, Gyeongsangnam-do, South Korea) of 5.5-6.5 months of age were slaughtered at a local abattoir, and uterus tissues were collected according to the follicular $(n=8)$ and luteal $(n=12)$ phases. The tissues were washed with phosphate-buffered saline (PBS) and fixed in 10\% formalin until analysis or stored at $-70^{\circ} \mathrm{C}$ for further experiments. The animal experiments were approved by the Pusan National University Institutional Animal Care and Use Committee.

Reverse transcription-quantitative polymerase chain reaction $(R T-q P C R)$. Total RNA was extracted using TRIzol reagent (Invitrogen; Thermo Fisher Scientific, Inc., Waltham, MA, USA) according to the manufacturer's protocol. The concentration of total RNA was measured by a spectrophotometer (Biospec-nano; Shimadzu, Kyoto, Japan). First-strand complementary DNA (cDNA) was prepared from total RNA $(3 \mu \mathrm{g})$ by RT using M-MLV reverse transcriptase (Invitrogen; Thermo Fisher Scientific, Inc.) and random primers (9-mers; Takara Bio, Inc., Otsu, Japan). RT-qPCR was performed with cDNA template $(2 \mu \mathrm{l})$ and $2 \mathrm{X}$ Power SYBR Green (6 $\mu \mathrm{l}$; Toyobo Co., Ltd., Osaka, Japan) containing specific primers. Primer sequences for $\beta$-actin, S100 calcium-binding protein G (S100G), ESR1, ESR2, PGR, LHCGR, OXT, OXTR, HPGD and GJA1 are presented in Table I. RT-qPCR was conducted for 40 cycles using the following parameters: Denaturation at $95^{\circ} \mathrm{C}$ for $15 \mathrm{sec}$, followed by annealing and extension at $70^{\circ} \mathrm{C}$ for $60 \mathrm{sec}$. Fluorescence intensity was measured at the end of the extension phase of each cycle. The threshold value for the fluorescence intensity of each sample was set manually. The reaction cycle at which PCR products exceeded this fluorescence intensity threshold during exponential phase of PCR amplification was considered to be the quantification cycle $(\mathrm{Cq})$. Expression of the target gene was quantified relative to that of $\beta$-actin, a ubiquitous housekeeping gene, based on comparison of Cqs at constant fluorescence intensity.

Western blotting analysis. Protein samples were extracted with PRO-PREP solution (iNtRON Biotechnology, Seoul, South Korea) following the manufacturer's protocol. A total of $30 \mu \mathrm{g}$ cytosolic proteins were separated by $8-12 \%$ SDS-polyacrylamide gel electrophoresis (SDS-PAGE) and transferred to nitrocellulose membranes (Daeillab Service Co., Ltd., Seoul, South Korea). Membranes were then blocked for $2 \mathrm{~h}$ with $5 \%$ skimmed milk (Difco; BD Biosciences, Sparks, MD, USA) in PBS with 0.05\% Tween-20 (PBS-T). Following blocking, membranes were incubated with ESR1 (1:500), ESR2 (1:500), PGR (1:500), LHCGR (1:500), OXTR 
Table I. Primer sequences for reverse transcription-quantitative polymerase chain reaction.

\begin{tabular}{|c|c|c|c|}
\hline Gene name & Primer & Sequence $\left(5^{\prime}-3^{\prime}\right)$ & Fragment size (bp) \\
\hline$\beta$-actin & $\begin{array}{l}\text { Forward } \\
\text { Reverse }\end{array}$ & $\begin{array}{l}\text { TCCCTGGAGAAGAGCTACGA } \\
\text { CGCACTTCATGATCGAGTTG }\end{array}$ & 149 \\
\hline S100G & $\begin{array}{l}\text { Forward } \\
\text { Reverse }\end{array}$ & $\begin{array}{l}\text { TCCTGCAGAACTGAAGAGCA } \\
\text { TCCATCTCCATTCTTGTCCA }\end{array}$ & 172 \\
\hline ESRl & $\begin{array}{l}\text { Forward } \\
\text { Reverse }\end{array}$ & $\begin{array}{l}\text { AGCACCCTGAAGTCTCTGGA } \\
\text { TGTGCCTGAAGTGAGACAGG }\end{array}$ & 160 \\
\hline ESR2 & $\begin{array}{l}\text { Forward } \\
\text { Reverse }\end{array}$ & $\begin{array}{l}\text { GTGATCACACAACCCGAGTG } \\
\text { ATGAAGCCCGGAATTTTCTT }\end{array}$ & 214 \\
\hline$P G R$ & $\begin{array}{l}\text { Forward } \\
\text { Reverse }\end{array}$ & $\begin{array}{l}\text { GATTCAGAAGCCAGCCAGAG } \\
\text { CGTCGTTTCTTCCAGCACA }\end{array}$ & 185 \\
\hline$L H C G R$ & $\begin{array}{l}\text { Forward } \\
\text { Reverse }\end{array}$ & $\begin{array}{l}\text { TCCGAAAGCTTCCAGATGTT } \\
\text { TGCATCTTCTTCAGGTGTGC }\end{array}$ & 238 \\
\hline$O X T$ & $\begin{array}{l}\text { Forward } \\
\text { Reverse }\end{array}$ & $\begin{array}{l}\text { CGCCTGCTACATCCAGAACT } \\
\text { CGGCAGGTAGTTCTCCTCCT }\end{array}$ & 193 \\
\hline OXTR & $\begin{array}{l}\text { Forward } \\
\text { Reverse }\end{array}$ & $\begin{array}{l}\text { TGCTACGGCCTTATCAGCTT } \\
\text { GCCTTGGAGATGAGCTTGAC }\end{array}$ & 158 \\
\hline$H P G D$ & $\begin{array}{l}\text { Forward } \\
\text { Reverse }\end{array}$ & $\begin{array}{l}\text { AAGGCGGCATCATTATCAAC } \\
\text { GCAAATGGCATTCAGTCTCA }\end{array}$ & 161 \\
\hline GJAl & $\begin{array}{l}\text { Forward } \\
\text { Reverse }\end{array}$ & $\begin{array}{l}\text { CACCAGGTGGACTGTTTCCT } \\
\text { TCTTTCCCTTCACACGATCC }\end{array}$ & 151 \\
\hline
\end{tabular}

bp, base pairs; S100G, S100 calcium-binding protein G; ESR1, estrogen receptor 1; ESR2, estrogen receptor 2; PGR, progesterone receptor; LHCGR, luteinizing hormone/choriogonadotropin receptor; OXT, oxytocin; OXTR, OXT receptor; HPGD, hydroxyprostaglandin dehydrogenase 15-(NAD); GJA1, gap junction $\alpha-1$ protein.

(1:500), HPGD (1:500) and GJA1 (1:1,000) overnight, followed by HRP-conjugated anti-rabbit and anti-goat secondary antibodies $(1: 2,000)$ in $5 \%$ skimmed milk with PBS-T for 1 h. Luminol reagent (Bio-Rad Laboratories, Inc., Hercules, CA, USA) was used to visualize antibody binding. Each blot was then stripped by incubation with $2 \%$ SDS and $100 \mathrm{mM}$ mercaptoethanol in $62.5 \mathrm{mM}$ Tris- $\mathrm{HCl}(\mathrm{pH} 6.8)$ for $30 \mathrm{~min}$ at $50-60^{\circ} \mathrm{C}$. Membranes were subsequently probed with antibody against $\beta$-actin $(1: 2,000)$ as an internal control. Blots were scanned using Gel Doc 1000, version 1.5 (Bio-Rad Laboratories, Inc.), and band intensities were normalized to $\beta$-actin levels.

Dot blot analysis. Total proteins prepared from porcine uterus tissues were transferred to a nitrocellulose membrane using a SlotBlot kit (Pharmacia Biotech, Inc., Piscataway Township, NJ, USA). The membrane was incubated with the antibody specific for OXT $(1: 5,000)$ for $1 \mathrm{~h}$, followed by the HRP-conjugated anti-mouse secondary antibody $(1: 2,000)$ in $5 \%$ skimmed milk with TBS-T for $1 \mathrm{~h}$. Membranes were subsequently probed with the antibody against $\beta$-actin $(1: 2,000)$ as an internal control. Blots were then visualized by Luminol reagent and analyzed by Gel Doc 1000 (Bio-Rad Laboratories, Inc.). Band intensities were normalized to $\beta$-actin levels.

Histological analysis. Uterus tissues were fixed with $10 \%$ formalin, embedded in paraffin wax, routinely processed, then sectioned into $4-\mu \mathrm{m}$-thick slices. Tissue sections were deparaffinized and rehydrated through graded alcohol using standard procedures and then stained with hematoxylin \& eosin (H\&E; Sigma-Aldrich; Merck Millipore). Images were captured at a magnification of $\mathrm{x} 40$ using a model BX50F-3 optical microscope (Olympus Corporation, Tokyo, Japan) and examined for histological analysis.

Statistical analysis. Results are presented as the mean \pm standard deviation. Data were analyzed using Sigma Plot, version 10.0 (Systat Software, Inc., San Jose, CA, USA). P<0.05 was considered to indicate a statistically significant difference.

\section{Results}

Preparation of porcine uterus tissue. In the first experiment, to distinguish the porcine uterus according to follicular and luteal phases, porcine uteruses were examined by histological analysis and luteal phase marker gene (Fig. 1). The tissues were fixed and stained with H\&E (Fig. 1A). Based on histological analysis by typical characteristics of estrous uterine endometrium, by observing a developed endometrial layer that was relatively thicker than that of the luteal phase, uteruses were divided into the follicular and luteal phases (Fig. 1B). Additionally, the transcriptional level of S100G, a biomarker of the luteal phase of porcine uterus, was observed (13). The transcriptional levels were increased up to 10 -fold in the luteal phase as predicted (Fig. 1C).

Transcriptional and translational expression levels of ESRI, ESR2, PGR, and LHCGR in the porcine uterus according to 
A Follicular phase

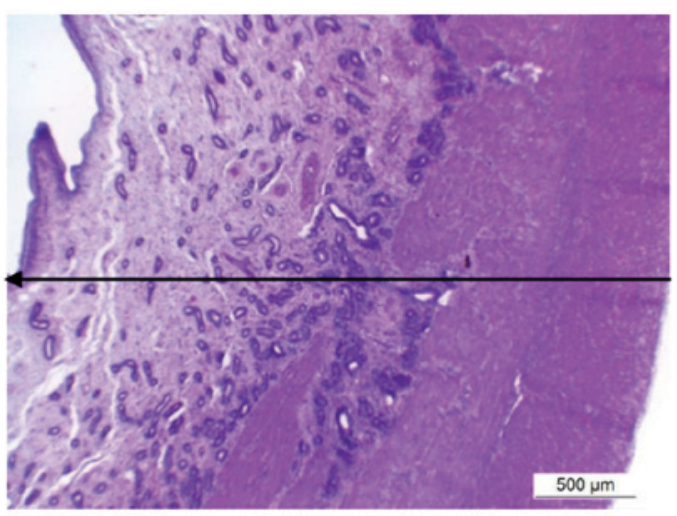

B

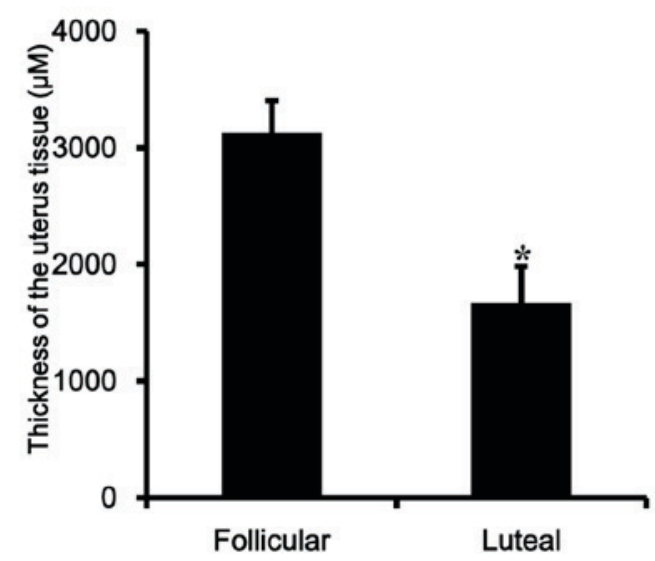

Luteal phase

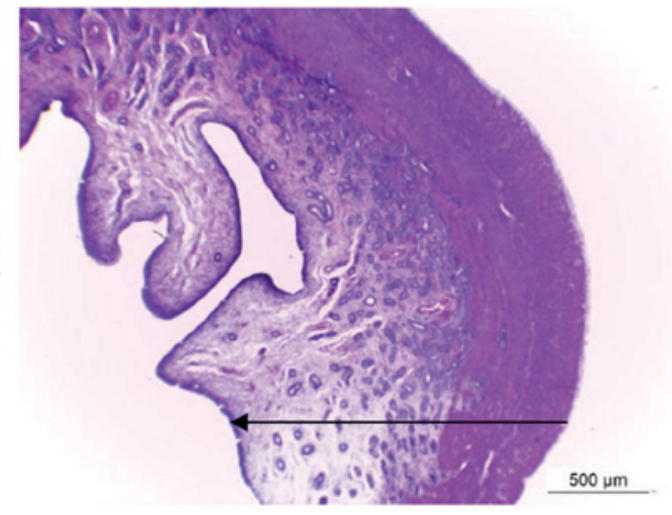

C

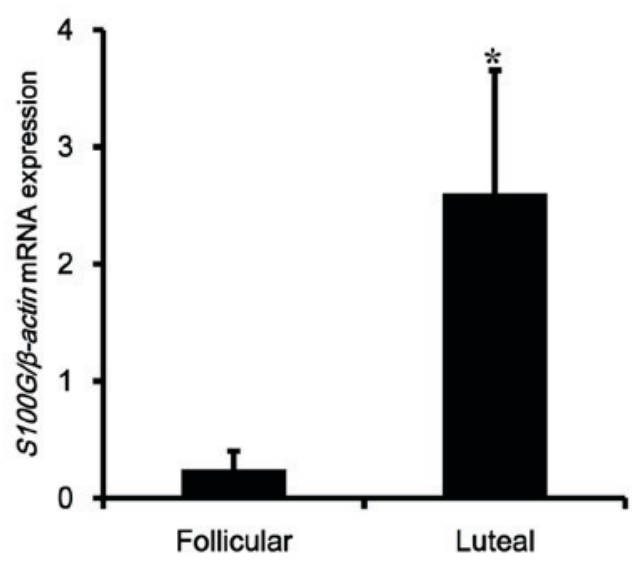

Figure 1. H\&E staining of porcine uterus tissues according to the estrous cycle. (A) Uterus tissues were stained with H\&E to confirm uterine morphology and thickness (black arrow) according to the estrous cycle (magnification, $\mathrm{x} 4$ ). (B) Quantification of thickness of uterus tissue according to the estrous cycle is indicated. (C) Expression levels of S100G mRNA were used as a biomarker of the luteal phase in the porcine uterus. Data were expressed as the mean \pm standard deviation from tissues of a minimum of 4 pigs. Gene expression was normalized to that of an internal control gene $\left(\beta\right.$-actin). ${ }^{*} \mathrm{P}<0.05$ vs. the follicular phase group. H\&E, hematoxylin and eosin; S100G, S100 calcium-binding protein G.

estrous cycle. To confirm regulation of contraction-associated factors according to the estrous cycle, mRNA and protein levels of reproductive hormonal receptors were examined. The expression levels of ESR1, ESR2, PGR and LHCGR mRNA were analyzed in the porcine uterus. Expression of ESR1, ESR2, PGR and LHCGR mRNA were observed in the uterus, and transcriptional levels of ESR1, ESR2 and PGR were significantly upregulated during the luteal phase compared with the follicular phase (Fig. 2A-C). In contrast, expression of LHCGR was significantly reduced in the luteal phase (Fig. 2D).

To confirm expression of these genes at the translational level in the porcine uterus according to the estrous cycle, western blotting analysis was conducted for ESR1, ESR2, PGR and LHCGR proteins (Fig. 3). Consistent with the mRNA results, protein expression levels of ESR1, ESR2 and PGR were enhanced during the luteal phase compared with the follicular phase, although the results for ESR1 and ESR2 were not significant (Fig. 3A-C). Similar to the mRNA results, the protein levels of LHCGR (Fig. 3D) were significantly reduced during the luteal phase in the porcine uterus.
Transcriptional and translational expression of CAPs in porcine uterus according to the estrous cycle. To examine regulation of CAPs depending on the estrous cycle, the mRNA expression levels of OXT-associated genes, including OXT and OXTR, were examined (Fig. 4A and B). Transcriptional levels of OXT and OXTR were upregulated in the luteal phase. The PGF metabolic enzyme HPGD exhibited no significant differences in mRNA expression depending on the estrous cycle (Fig. 4C). Among the tested genes, mRNA expression of GJA1 was upregulated by 4-fold in the luteal phase compared with the follicular phase (Fig. 4D).

Subsequently, protein expression of CAPs, including OXTR, HPGD and GJA1 were examined during the follicular and luteal phases of the porcine uterus by western blot analyses. In addition, a dot-blot assay was conducted in order to detect the signal of OXT, due to its low molecular weight (Fig. 5). Protein expression patterns of OXT and GJA1 were similar to their mRNA levels, which were significantly upregulated during the luteal phase compared with the follicular phase (Fig. 5A and D). However, protein expression levels of OXTR and HPGD were different from the transcription results (Fig. 5B and C). Although mRNA levels of OXTR and 
A

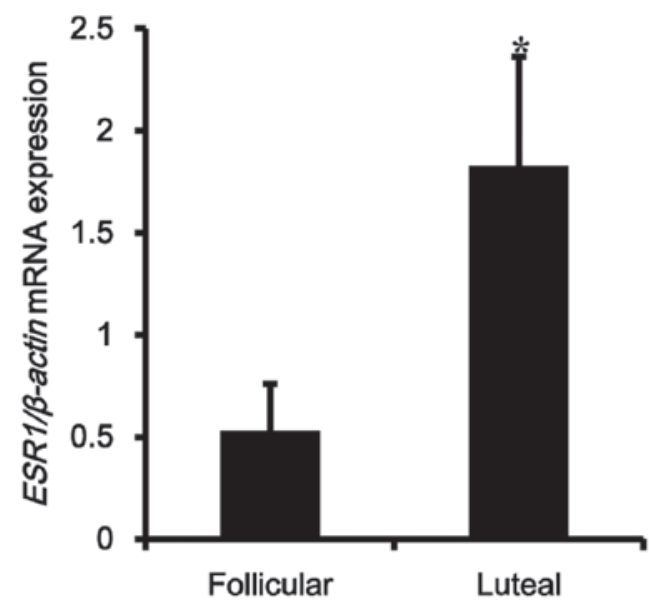

C

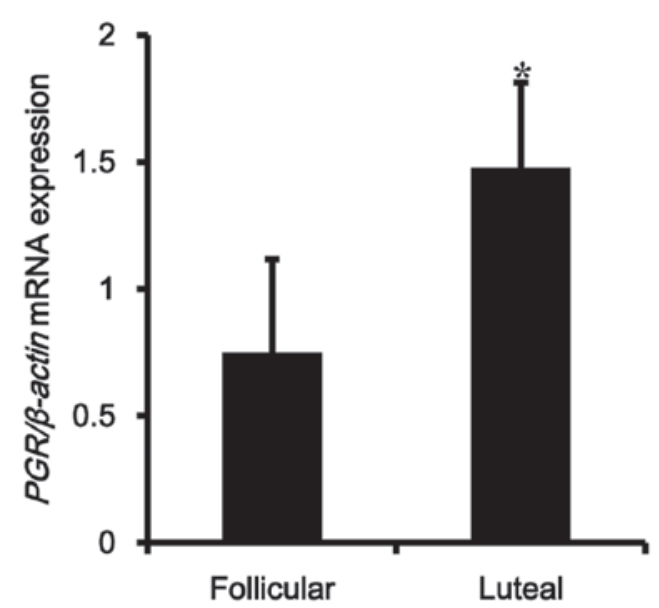

B

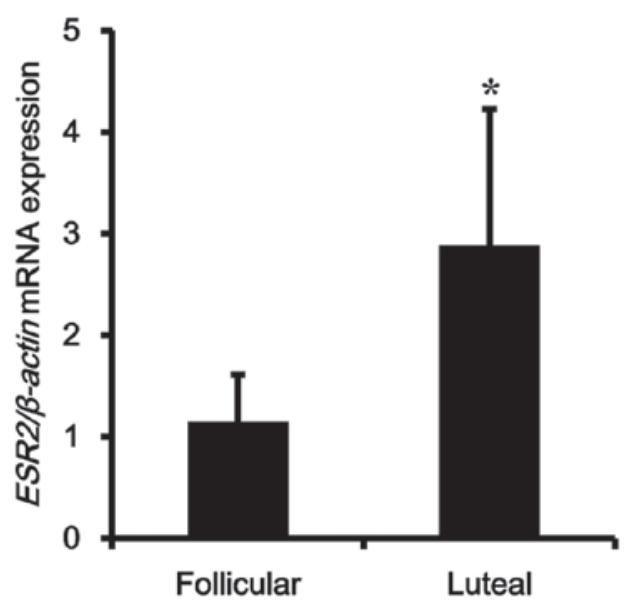

D

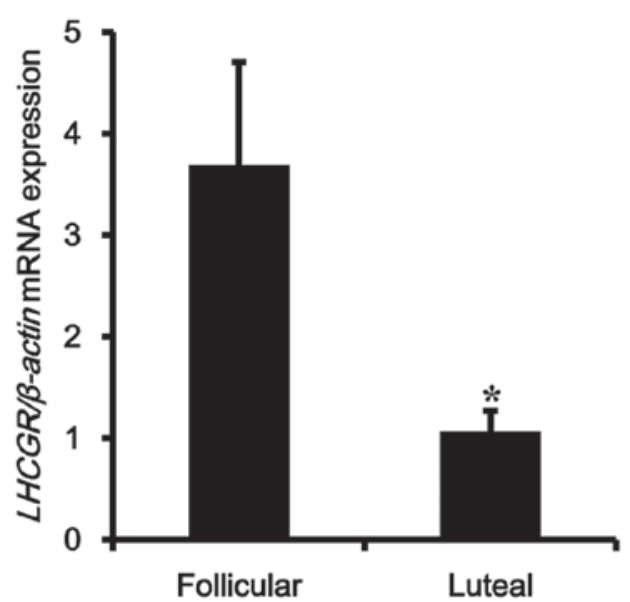

Figure 2. Transcriptional expression of ESR1, ESR2, PGR, and LHCGR in the porcine uterus according to the estrous cycle. Reverse transcription-quantitative polymerase chain reaction analysis verified the transcriptional levels of (A) ESR1, (B) ESR2, (C) PGR, and (D) LHCGR in the porcine uterus according to the follicular and luteal phases. Data were expressed as the mean \pm standard deviation from tissues of a minimum of 4 pigs. Gene expression was normalized to that of an internal control gene $\left(\beta\right.$-actin). ${ }^{*} \mathrm{P}<0.05$ vs. the follicular phase group. ESR, estrogen receptor; PGR, progesterone receptor; LHCGR, luteinizing hormone/choriogonadotropin receptor.

HPGD were augmented in the luteal phase, protein levels were observed to decline in the luteal phase.

\section{Discussion}

Study of the porcine reproductive system is important, due to the fact that pigs breed only twice a year, and pork consumption is increasing. The porcine estrous cycle spans a period of 18-24 days and consists of an follicular phase of 5-7 days and a luteal phase of 13-15 days (3). Following ovulation, the developing corpora lutea produces an increasing amount of $\mathrm{P} 4$, reaching peak concentrations 8-9 days after ovulation (14). Subsequently, luteolysis occurs around 15 days after ovulation if pigs are not pregnant in the luteal phase.

In the present study, the histological patterns of the uterus were examined in order to determine the follicular and luteal phases in the porcine uterus. In addition, examination of S100G expression confirmed the correct classification of porcine uterine tissues according to the estrous cycle. S100G is a cytosolic calcium-binding protein that is expressed predominantly in the duodenum, placenta and uterus. Although few studies have investigated the estrous cycle in the porcine uterus, regulation of the $\mathrm{S} 100 \mathrm{G}$ gene during the porcine estrous cycle has been investigated in a previous study, indicating that the S100G gene is a useful marker gene for the luteal phase of the porcine uterus (13).

Understanding the physiological characteristics of the uterus depending on concentrations of endogenous hormones and the estrous cycle is critically important due to the fact it contributes to enhanced livestock production, and physiological studies of this nature are currently lacking. A previous study observed that plasma levels of E2 were highly regulated during the follicular phase in pigs (15). In another study, P4 concentration in the luteal phase increased gradually during formation of the corpus luteum throughout the porcine estrous cycle (16).

In the present study, ESR1, ESR2, PGR and LHCGR were all expressed in the porcine uterus. Transcriptional and translational levels of ESR1, ESR2, and PGR were elevated during luteal phase compared to follicular phase, although alteration 

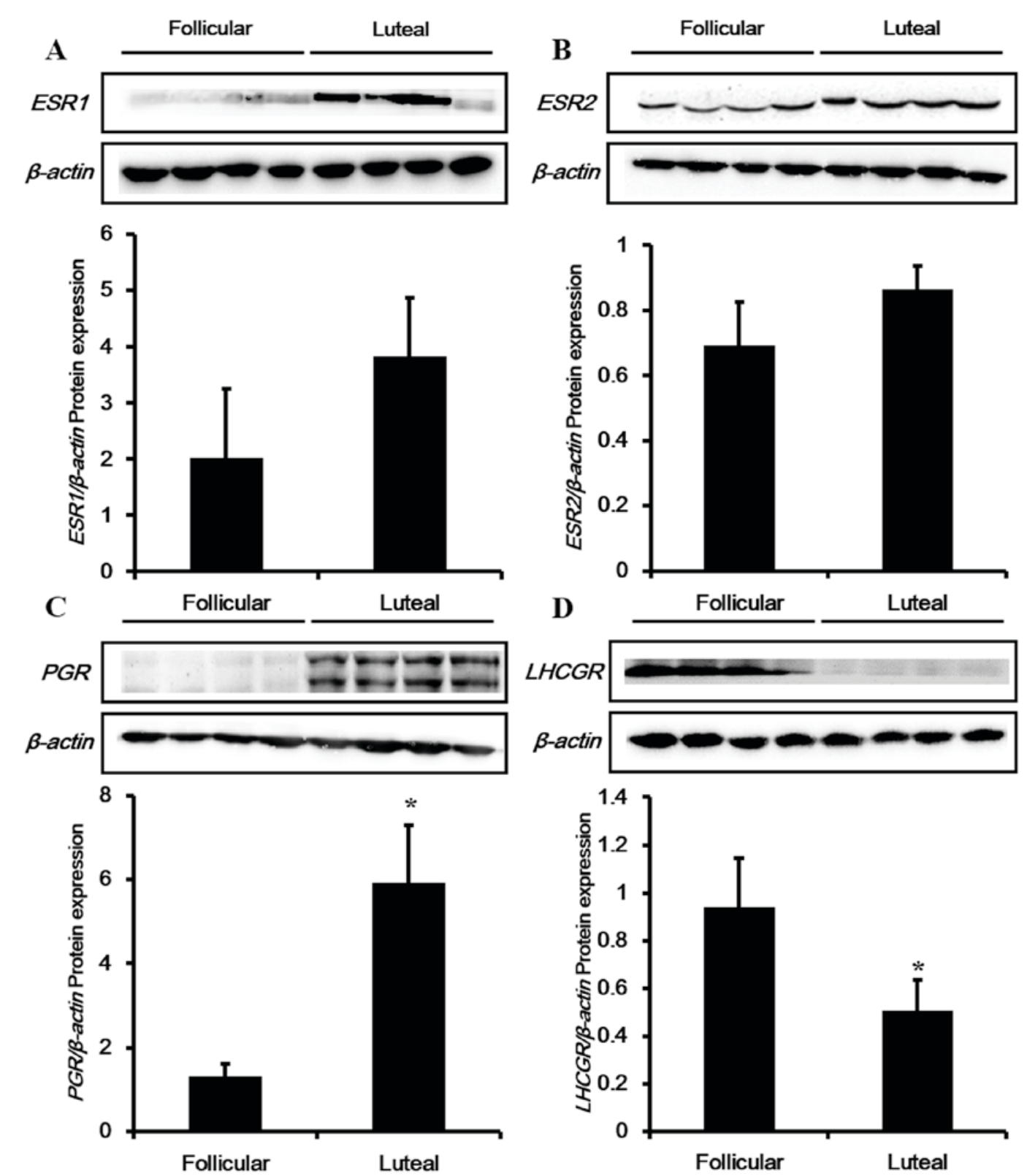

Figure 3. Translational expression of ESR1, ESR2, PGR, and LHCGR in the porcine uterus according to the estrous cycle. Western blot analysis verified the translational levels of (A) ESR1, (B) ESR2, (C) PGR, and (D) LHCGR in the porcine uterus according to the follicular and luteal phases. Data were expressed as the mean \pm standard deviation from tissues from a minimum of 4 pigs per group. Gene expression was normalized to that of an internal control protein ( $\beta$-actin). " $\mathrm{P}<0.05$ vs. the follicular phase group. ESR, estrogen receptor; PGR, progesterone receptor; LHCGR, luteinizing hormone/choriogonadotropin receptor.

of ESR1 and ESR2 protein expression was not significant. Both mRNA and protein expression levels of LHCGR were significantly reduced in the luteal phase. These results indicate that signaling of E2, P4 and LH can be dynamically regulated via expression of their cognitive receptors. Furthermore, it was previously reported that E2 maintains a high concentration during the follicular phase, whereas LH is highly produced in the luteal phase (17). Thereby, it is possible that regulation of ESR1, regulation of ESR1, ESR2 and LHCGR is under the negative feedback influence of their agonists, E2 and LH.

To the best of our current knowledge, only previous study has investigated the regulation of steroid receptor during the estrous cycle. Sukjumlong et al (18), examined the mRNA expression levels of ESR1, ESR2 and PGR in the uterine endometrium and myometrium of cyclic sows. It was identified that the level of ESR1 mRNA in the endometrium exhibited no significant differences depending on the estrous cycle, although ESR1 mRNA expression was greater in the follicular phase compared with the luteal phase in the myometrium. In the case of ESR2, mRNA expression was low in all stages with no significant difference depending on the estrous cycle. The mRNA level of PGR in follicular phase was higher than in the luteal phase in the sow uterus (18). However, these experiments were limited to only mRNA measurements, these results differ from those of the current study due to the distinct environmental conditions for pigs used. 
A

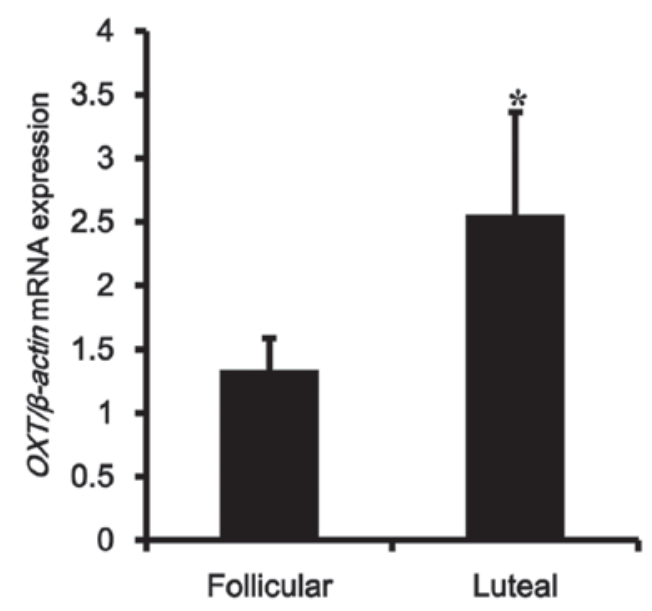

C

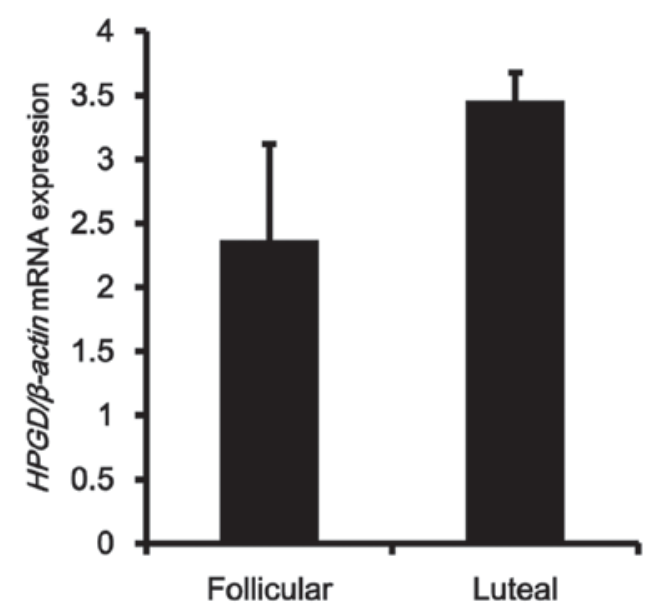

B

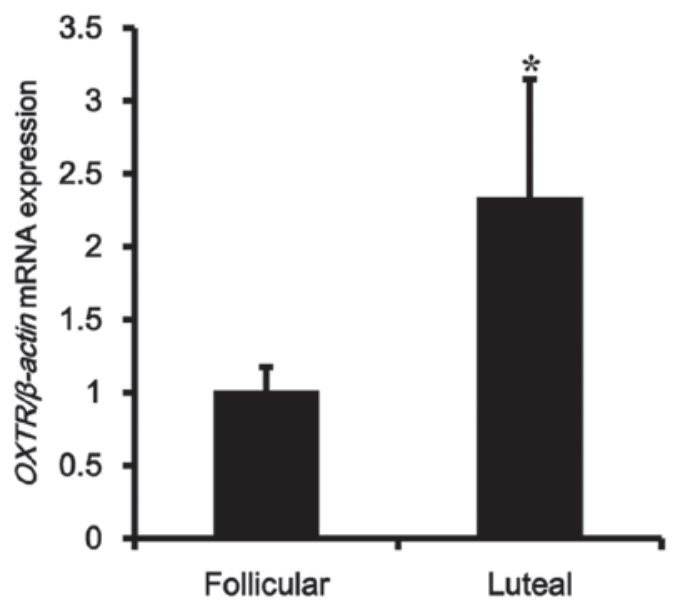

D

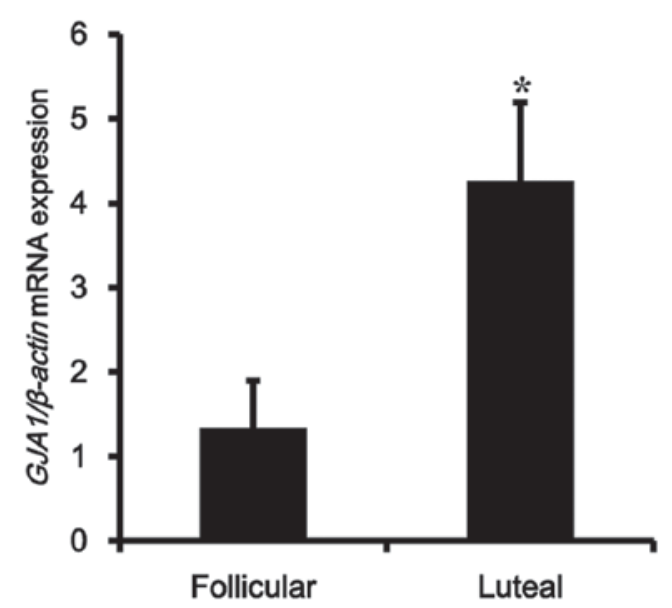

Figure 4. Transcriptional expression of contraction-associated proteins in the porcine uterus according to the estrous cycle. Reverse transcription-quantitative polymerase chain reaction analysis verified transcriptional levels of (A) OXT, (B) OXTR, (C) HPGD, and (D) GJA1 in the porcine uterus according to the follicular and luteal phases. Data were expressed as the mean \pm standard deviation from tissues from a minimum of 4 pigs. Gene expression was normalized to that of an internal control gene ( $\beta$-actin). ${ }^{*} \mathrm{P}<0.05$ vs. the follicular phase group. OXT, oxytocin; OXTR, OXT receptor; HPGD, hydroxyprostaglandin dehydrogenase 15-(NAD); GJA1, gap junction $\alpha-1$ protein.

In the uterus, regulation of myometrial contractility is regulated by expression of genes encoding CAPs, which is under the control of E2 and P4. Since expression of ESRs and PGR was differentially regulated during the estrous cycle, it was hypothesized that CAPs as indicators of uterine contractile activity may also be regulated during the estrous cycle. In order to confirm this hypothesis, mRNA and protein expression levels of CAPs were evaluated in the non-pregnant stage of the porcine uterus according to the estrous cycle. OXT and OXTR mRNA expression levels were observed to be significantly increased during the luteal phase compared with the follicular phase, although protein levels of OXTR were marginally reduced. At present, there is insufficient evidence available regarding regulation of OXT and OXTR in the uterus. In experimental animals, E2 was demonstrated to increase OXTR in the uterus of non-pregnant and pregnant rats (19-21).

HPGD, which oxidizes and inactivates PG at the 15-hydroxyl group, was expressed in the porcine uterus, and its mRNA levels exhibited no significant differences depending on the estrous cycle. However, protein expression of HPGD significantly increased during the follicular phase compared with the luteal phase. It was reported that E2 increases activity of HPGD in the uteruses of rats and humans $(22,23)$. In the present study, GJA1, a transmembrane and gap junction protein that serves an important role in contraction of muscle tissues, was identified to be expressed in the porcine uterus, and its mRNA level was significantly increased during the luteal phase compared with the follicular phase. Consistent with the mRNA results, protein expression of GJA1 was enhanced during the luteal phase. Although different tissue was tested, protein expression of GJA1 in the bovine corpus luteum was highest in the early luteal phase and decreased as the estrous cycle progressed (24). In summary, expression levels of specific receptors for E2, P4 and LH were observed together with CAPs in the porcine uterus during the estrous cycle. The results indicated that these proteins are dynamically regulated during the estrous cycle and may serve critical roles in the physiological function and contraction of the uterus. 

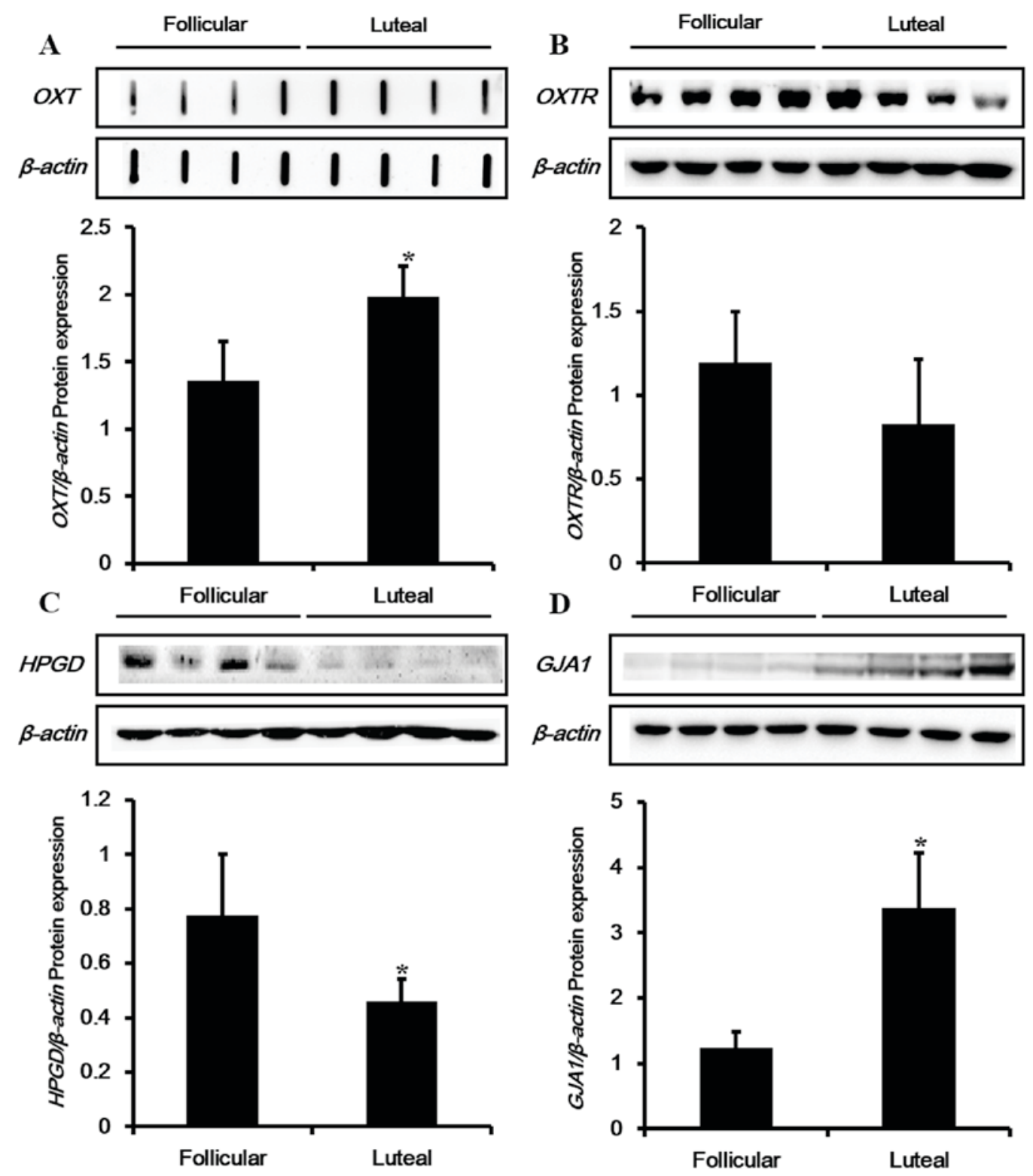

Figure 5. Translational expression of contraction-associated proteins in the porcine uterus according to the estrous cycle. (A) Dot blot analysis verified translational levels of OXT. Western blot analysis verified (B) OXTR, (C) HPGD, and (D) GJA1 in the porcine uterus according to the follicular and luteal phases. Data were expressed as the mean \pm standard deviation from tissues of a minimum of 4 pigs per group. Gene expression was normalized to that of an internal control protein ( $\beta$-actin). ${ }^{*} \mathrm{P}<0.05$ vs. the follicular phase group. OXT, oxytocin; OXTR, OXT receptor; HPGD, hydroxyprostaglandin dehydrogenase 15-(NAD); GJA1, gap junction $\alpha-1$ protein.

\section{Acknowledgements}

The current study was supported by the National Research Foundation of Korea grant funded by the Korea government (MEST; grant no. 2014R1A1A2057387).

\section{References}

1. An BS, Ahn HJ, Kang HS, Jung EM, Yang H, Hong EJ and Jeung EB: Effects of estrogen and estrogenic compounds, 4-tert-octylphenol, and bisphenol A on the uterine contraction and contraction-associated proteins in rats. Mol Cell Endocrinol 375: 27-34, 2013.

2. Dawe ST, Husband AJ and Langford CM: Effects of induction of parturition in ewes with dexamethasone or oestrogen on concentrations of immunoglobulins in colostrum, and absorption of immunoglobulins by lambs. Aust J Biol Sci 35: 223-230, 1982.
3. Soede N, Langendijk P and Kemp B: Reproductive cycles in pigs. Anim Reprod Sci 124: 251-258, 2011.

4. Bullivant SB, Sellergren SA, Stern K, Spencer NA, Jacob S Mennella JA and McClintock MK: Women's sexual experience during the menstrual cycle: Identification of the sexual phase by noninvasive measurement of luteinizing hormone. J Sex Res 41: 82-93, 2004.

5. Rzucidlo S, Weigl R and Tilton J: Myometrial LH/hCG receptors during the estrous cycle and pregnancy in pigs. Anim Reprod Sci 51: 249-257, 1998.

6. Krzymowski T and Stefańczyk-Krzymowska S: The oestrous cycle and early pregnancy-a new concept of local endocrine regulation. Vet J 168: 285-296, 2004.

7. Rush RW, Keirse MJ, Howat P, Baum JD, Anderson AB and Turnbull AC: Contribution of preterm delivery to perinatal mortality. Br Med J 2: 965-968, 1976.

8. López Bernal A, Rivera J, Europe-Finner GN, Phaneuf S and Asbóth G: Parturition: Activation of stimulatory pathways or loss of uterine quiescence? Adv Exp Med Biol 395: 435-451, 1994. 
9. Wu WX, Ma XH, Coksaygan T, Chakrabarty K, Collins V, Rose J and Nathanielsz PW: Prostaglandin mediates premature delivery in pregnant sheep induced by estradiol at 121 days of gestational age. Endocrinology 145: 1444-1452, 2004.

10. Csapo AL, Knobil E, Van Der Molen HJ and Wiest WG: Peripheral plasma progesterone levels during human pregnancy and labor. Am J Obstet Gynecol 110: 630-632, 1971.

11. Mesiano S and Welsh TN: Steroid hormone control of myometrial contractility and parturition. Seminars in cell \& developmental biology 18: 321-331, 2007.

12. Renthal NE, Chen CC, Williams KC, Gerard RD, Prange-Kiel J and Mendelson CR: miR-200 family and targets, ZEB1 and ZEB2, modulate uterine quiescence and contractility during pregnancy and labor. Proc Natl Acad Sci USA 107: 20828-20833, 2010.

13. Yun SM, Choi KC, Kim IH, An BS, Lee GS, Hong EJ, Oh GT and Jeung EB: Dominant expression of porcine Calbindin-D9k in the uterus during a luteal phase. Mol Reprod Dev 67: 251-256, 2004

14. Knox R: Recruitment and selection of ovarian follicles for determination of ovulation rate in the pig. Domest Anim Endocrinol 29: 385-397, 2005.

15. Sukjumlong S, Kaeoket K, Dalin AM and Persson E: Immunohistochemical Studies on Oestrogen Receptor Alpha (ER Alpha) and the Proliferative Marker Ki-67 in the Sow Uterus at Different Stages of the Oestrous Cycle. Reprod Domest Anim 38: 5-12, 2003.

16. Ribeiro LA, Bacci ML, Seren E, Tamanini C and Forni M: Characterization and differential expression of vascular endothelial growth factor isoforms and receptors in swine corpus luteum throughout estrous cycle. Mol Reprod Dev 74: 163-171, 2007.
17. Cassidy A, Bingham S and Setchell KD: Biological effects of a diet of soy protein rich in isoflavones on the menstrual cycle of premenopausal women. Am J Clin Nutr 60: 333-340, 1994.

18. Sukjumlong S, Persson E, Dalin AM, Janson V and Sahlin L: Messenger RNA levels of estrogen receptors alpha and beta and progesterone receptors in the cyclic and inseminated/early pregnant sow uterus. Anim Reprod Sci 112: 215-228, 2009.

19. Murata T, Narita K, Honda K and Higuchi T: Changes of receptor mRNAs for oxytocin and estrogen during the estrous cycle in rat uterus. J Vet Med Sci 65: 707-712, 2003.

20. Murata T, Narita K, Honda K, Matsukawa S and Higuchi T: Differential regulation of estrogen receptor alpha and beta mRNAs in the rat uterus during pregnancy and labor: Possible involvement of estrogen receptors in oxytocin receptor regulation. Endocr J 50: 579-587, 2003.

21. Murata T, Murata E, Liu C, Narita K, Honda K and Higuchi T: Oxytocin receptor gene expression in rat uterus: Regulation by ovarian steroids. J Endocrinol 166: 45-52, 2000.

22. Matsuo M, Ensor CM and Tai HH: Characterization of the genomic structure and promoter of the mouse NAD+-dependent 15-hydroxyprostaglandin dehydrogenase gene. Biochem Biophys Res Commun 235: 582-586, 1997.

23. Kelly RW, Linan C, Thong J, Yong EL and Baird DT: Prostaglandin inactivation is increased in endometrium after exposure to clomiphene. Prostaglandins Leukot Essent Fatty Acids 50: 235-238, 1994.

24. Grazul-Bilska AT, Redmer DA, Johnson ML, Jablonka-Shariff A, Bilski JJ and Reynolds LP: Gap junctional protein connexin 43 in bovine corpora lutea throughout the estrous cycle. Biol Rep 54: 1279-1287, 1996 